\title{
Infective Endocarditis in a Patient with Ventricular Septal Defect and Patent Ductus Arteriosus
}

\author{
Prenali Dwisthi Sattwika ${ }^{1,{ }^{*}}$, Anggoro Budi Hartopo ${ }^{1,2}$, Dyah Wulan Anggrahini ${ }^{2}$, Hasanah Mumpuni ${ }^{1,2}$ \\ ${ }^{1}$ Department of Internal Medicine, Faculty of Medicine, Universitas Gadjah Mada-Dr. Sardjito Hospital, ${ }^{1}$ Department of \\ Cardiology and Vascular Medicine, Faculty of Medicine, Universitas Gadjah Mada-Dr. Sardjito Hospital, Yogyakarta, \\ Indonesia \\ *correspondence: pdsattwika@yahoo.com
}

\begin{abstract}
Introduction: Congenital heart disease is a predisposing factor for infective endocarditis (IE). We herein experienced a case of IE with concurrent left to right shunt ventricular septal defect (VSD) and patent ductus arteriosus (PDA).

Case Presentation: A 26-year-old woman with a history of dental problem presented with a chief complain of worsening dyspnea over the past one month. She developed multiple petechiae over her extremities. Physical examination revealed cardiomegaly, a grade 3/6 pansistolic murmur heard at the lower left sternal edge, and systolic ejection murmur heard in the pulmonary area. Her hemoglobin level was $5.8 \mathrm{~g} / \mathrm{dl}$ with thrombocyte count of $59 \times 10^{3} / \mathrm{\mu L}$. A transthoracal echocardiography (TTE) revealed a perimembranous VSD, PDA, moderate to severe tricuspid regurgitation, and pulmonary hypertension with ejection fraction of $78 \%$. Multiple vegetations of size $1.4 \times 0.9 \mathrm{~cm}$ were attached to the pulmonal valve. She was started on intravenous ampicillin-sulbactam 3 gram 6 hourly and gentamycin $120 \mathrm{mg} 24$ hourly. She also received sildenafil and furosemide. Streptococcus viridans was identified in blood culture from the right hand, Streptococcus porcinus from the right leg, and Streptococcus mitis/oralis from the left leg. TTE evaluation showed persistence of the vegetation and new vegetation of size $1 \times 1 \mathrm{~cm}$ attached to the VSD with negative blood culture.

Discussion: This case illustrates a loss to follow-up congenital heart disease that eventually develops multi-pathogen IE. The patient showed no clinical stigmata of IE, but presented with bicytopenia. Increase in blood flow through the pulmonary artery and septal defect may result in endothelial damage that facilitates vegetation formation. She is planned for open heart surgery to evacuate the vegetations.
\end{abstract}

Conclusion: Our cases emphasizes the significance of careful assessment for IE in individual with congenital heart disease to reach an optimal outcome.

Keywords: Infective endocarditis; ventricular septal defect; patent ductus arteriosus 